Hautarzt $2017 \cdot 68: 518-519$

DOI 10.1007/s00105-017-4007-3

Online publiziert: 19. Juni 2017

(c) Springer Medizin Verlag GmbH 2017

CrossMark

\author{
C. Rose ${ }^{1} \cdot$ E. von Stebut ${ }^{2,3}$ \\ 'Dermatopathologie Lübeck, Lübeck, Deutschland \\ ${ }^{2}$ Hautklinik, Universitätsmedizin Mainz, Johannes Gutenberg-Universität Mainz, Mainz, Deutschland \\ ${ }^{3}$ Mainz Research School of Translational Biomedicine (TransMed), Universitätsmedizin Mainz, Johannes \\ Gutenberg-Universität Mainz, Mainz, Deutschland
}

\title{
Granulomatöse Erkrankungen der Haut
}

\section{Stets eine besondere Herausforderung}

Liebe Kolleginnen und Kollegen!

Granulomatöse Erkrankungen definieren sich durch das feingewebliche Korrelat einer granulomatösen Entzündung, und daraus folgt unmittelbar, dass es zur Diagnosestellung einer Gewebsentnahme zur histologischen Untersuchung bedarf. Kein weiteres Organ ist dabei so einfach zugänglich wie die äußere Haut. Der erste Schritt der Zuordnung zur Gesamtgruppe der granulomatösen Erkrankungen ist für den Dermatopathologen in der Regel recht einfach. Dann jedoch - wesentlich schwieriger - ist der zweite Schritt der genauen Diagnosestellung und die sichere Abgrenzung zu den Differenzialdiagnosen. Es bedarf einer sorgfältigen klinisch-pathologischen Korrelation unter Einbeziehung verschiedener Fachgebiete und des Abgleichs mit paraklinischen Befunden. Gelegentlich lässt sich anfangs nur eine deskriptive Arbeitsdiagnose formulieren, und erst im Krankheitsverlauf wird die exakte Diagnose klar.

\section{》) Für die Diagnosestellung ist eine Gewebsentnahme zur histologischen Untersuchung notwendig}

Granulomatöse Hautinfiltrate manifestieren sich klinisch oft als nicht schuppende Knoten und Plaques, die auf Glasspateldruck ein apfelgeleeartiges bzw. rehbraunes Eigeninfiltrat erkennen lassen. Überraschenderweise wurde die Bedeutung dieses einfachen Verfahrens für die Dermatologie erst am Ende des 19. Jahrhunderts entdeckt. Die Bezeichnung der Untersuchung als Diaskopie geht auf den Hamburger Dermatologen Paul Gerson Unna zurück, der 1893 die Vorteile der Untersuchung mittels Glasdruck beschrieb. Zwei Jahre zuvor war die Untersuchung der Haut mittels Glasdruck vom Berliner Pharmakologen Liebreich in einem Vortrag vorgestellt und anschließend publiziert worden. Unna räumte später die Erstbeschreibung von Liebreich klar ein, ohne ihn aber in Folgepublikationen zu erwähnen. Die genauen historischen Zusammenhänge dieses Streits werden von Schönfeld in einem kleinen lesenswerten Beitrag aus dem Jahr 1957 dargestellt [1]. Der heutige Leser schmunzelt.

Erstmals widmet sich nun ein gesamtes Leitthemenheft der Zeitschrift Der Hautarzt in 6 Beiträgen dieser spannenden Krankheitsgruppe. Die Beiträge spannen dabei einen weiten Bogen von den immunologischen Grundlagen der Granulomentstehung über die Schwierigkeiten der histopathologischen Diagnostik bis zur ausführlichen Darstellung verschiedener spezieller Krankheitsbilder.

In der Eingangsarbeit beantwortet Esther von Stebut die kurze Frage: „Was ist ein Granulom?" Die Antwort ist hochinteressant und spannend zu lesen. In letzter Zeit konnten neue Erkenntnisse aus experimentellen Arbeiten an Tiermodellen, aber auch aus klinischen
Beobachtungen nach Anwendung moderner Biologika, deren bekannte $\mathrm{Ne}$ benwirkung die Reaktivierung einer Tuberkulose sein kann, gewonnen werden.

Tina Giner hat mit Kollegen der Würzburger Universitäts-Hautklinik eine sehr klare und hervorragend bebilderte Übersicht zur Sarkoidose beigesteuert. Sehr hilfreich für den Leser dürften auch die übersichtlichen Tabellen zum diagnostischen Vorgehen und den Differenzialdiagnosen sowie die grafische Darstellung des Therapiealgorithmus sein.

Beate Weidenthaler-Barth stellt in einer Übersicht das Spektrum der Palisadengranulome dar und geht dabei auf die Klinik, die Histopathologie sowie kurz auf therapeutische Optionen ein.

Interessanterweise treten einige granulomatöse Erkrankungen, wie z. B. der Lupus pernio oder die lupoide Rosazea vorzugsweise im Gesicht auf. Florian Butsch bespricht systematisch die verschiedenen Krankheitsbilder und geht dabei auch auf das Xanthogranulom und das Granuloma eosinophilicum faciei (Granuloma faciale) ein. Obwohl der Name eine granulomatöse Erkrankung suggeriert, handelt es sich aber im ersten Fall um eine Nicht-Langerhans-ZellHistiozytose und bei Letzterem um eine lokalisierte Variante einer leukozytoklastischen Vaskulitis. Derartige historisch bedingte Fehlbezeichnungen mögen mit ein Grund sein, wieso die Dermatologie bei Studenten oft als unübersichtliches Fach angesehen wird. 
Deutschland hat in den letzten Jahren vermehrt Kriegsflüchtlingen aus Endemiegebieten der Leishmaniose, wie z. B. Syrien und Afghanistan, Zuflucht geboten. Hierdurch wird diese in den Praxen und Kliniken häufiger gesehen. Esther von Stebut fasst in ihrem zweiten Beitrag alles Wesentliche einschließlich der Therapieoptionen dieser wichtigen infektiösen Hauterkrankung zusammen.

Im letzten Artikel von Konstanze HollUlrich, der Leiterin des Konsultationsund Referenzzentrums für VaskulitisDiagnostik, und Christian Rose geht es um besondere granulomatöse Reaktionsmuster in der Haut. Unter anderem wird beantwortet, welche Diagnosen zu beachten sind, wenn histologisch eine granulomatöse Entzündung in Kombination mit einer Vaskulitis gefunden wird.

Auch wenn die Beiträge nicht alle Aspekte dieser großen Krankheitsgruppe umfassend darstellen können, so hoffen wir doch, dass Sie einige neue und interessante Erkenntnisse gewinnen können.

Ihre

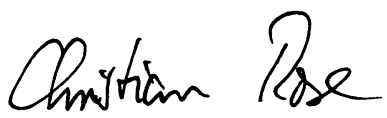

Christian Rose

\section{Esther wou Stoont}

Esther von Stebut

\section{Korrespondenzadresse}

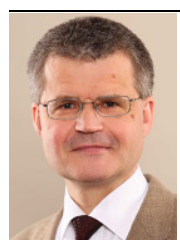

Priv.-Doz. Dr. C. Rose

Dermatopathologie Lübeck

Maria-Goeppert-Str. 5,

23562 Lübeck, Deutschland

rose@dermatohistologie-

luebeck.de

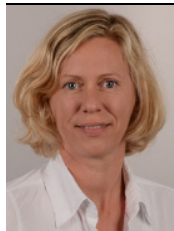

Univ.-Prof. Dr. E. von Stebut

Hautklinik, Universitäts-

medizin Mainz, Johannes

Gutenberg-Universität Mainz

Langenbeckstr. 1,

55131 Mainz, Deutschland

vonstebu@mail.uni-mainz.de

Interessenkonflikt. C. Rose und E. von Stebut geben an, dass kein Interessenkonflikt besteht.

\section{Literatur}

1. Schönfeld W (1957) Das Alter von "Lupe" und "Diaskopie" in der dermatologischen Diagnostik. Dermatol Wochenschr 136:1231-1235
Personalisierte Medizin in der Onkologie

"Krebs ist immer eine Erkrankung der Gene". Diese Aussage wird mittlerweile von allen Fachdisziplinen der Medizin akzeptiert. Bei den meisten Patienten

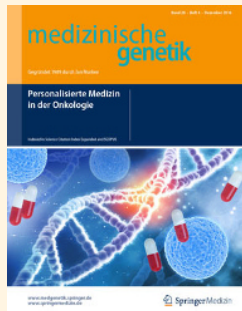
sind dabei die genetischen und auch epigenetischen Veränderungen somatisch erworben und auf die Tumorzellen beschränkt. Deshalb sind die

Aspekte der somatischen Humangenetik von überragender Bedeutung für die Onkologie.

In Ausgabe 4/16 der Zeitschrift medizinische genetik können Sie den aktuellen Status und zukünftige Entwicklungen im Bereich der personalisierten Medizin in diesen Beiträgen verfolgen:

- Internationales Krebsgenomkonsortium (ICGC)

- Das Tumorepigenom von der Genregulation über die Tumorklassifikation zum Therapietarget

- Personalisierte Medizin in der Hämatologie am Beispiel der akuten myeloischen Leukämie

- Personalisierte Medizin bei soliden Tumoren

- Personalisierte Medizin: Strukturen, Tumorboards, Visionen

- Soziale und ethische Aspekte der personalisierten Medizin

Bestellen Sie diese Ausgabe zum Preis von 36,- EUR zzgl. Versandkosten bei Springer Customer Service Center, Kundenservice Zeitschriften

Tel.: +49 6221-345-4303

E-Mail: leserservice@springer.com

\section{Suchen Sie noch mehr zum Thema?} Mit e.Med - dem Kombi-Abo von Springer Medizin - können Sie schnell und komfortabel in über 600 medizinischen Fachzeitschriften recherchieren und auf alle Inhalte im Volltext zugreifen. 